\title{
Theoretical Basis of Nanobioelectronics
}

\author{
Victor Lakhno ${ }^{1, \star}$ \\ ${ }^{1}$ Institute of Mathematical Problems of Biology RAS - the Branch of Keldysh Institute of Applied Mathematics \\ of RAS, 142290, Pushchino, Moscow Region, Russia
}

\begin{abstract}
Several years ago, the modern silicon-based nanoelectronics came to the limits of its miniaturization. Its advancement into the range of $10 \mathrm{~nm}$ and less faces enormous challenges. A breakthrough in further miniaturization of electronic devices can be realized only on the basis of new approaches of which nanobioelectronics seems to be the most promising.
\end{abstract}

\section{Introduction. DNA is a basis for the construction of electronic devices}

Nanobioelectronics relies on the use of charge transfer processes in biomacromolecules and molecular structures of nanometer size based thereon.

The main areas of nanobioelectronics are development of biosensors, complex nanoelectronic DNA-based schemes, construction of nanobiotransistors, nanomotors, nanorobots, etc.

This review presents theoretical and practical approaches to the development of the "hardware components" of bionanoelectronics - DNA-based nanowires, electronic sensors, memory devices, and logical elements. DNA is the only molecule which can reproduce itself. DNA can be used for creating complex structures and chains out of which nanobioelectronic devices can be constructed [1-3].

One can insert various complexes and clusters into the spatial nanoconstructions obtained so that they be arranged at strictly fixed distances from one another. In such a way one can crystallize there such compounds which are not crystallized in normal conditions.

Complex structures produced from DNA can be selectively metallized. This can be achieved by adding special enzymes into a solution. These enzymes identify special sequences of bases and attach themselves to them, thus shielding these regions from metallization by metal ions in a solution. On completing the metallization procedure enzymes can be separated from DNA by biochemical methods. In this way one gets predetermined metallized and nonmetallized regions of complex DNA structures [4]. A great impetus for constructing DNA-based electronic devices was the discovery that DNA has conductive properties.

In section 2 we will give a short review of the main experiments on the charge transfer and DNA conducting properties. In section 3 we will deal with the methods of constructing DNA-based nanowires. In section 4 we discuss the polaron mechanism of charge transfer in DNA. In section 5 we discuss a possibility of creating nanobiochips which use unique conducting properties of DNA. Section 6 is devoted to DNA-based electronic memory devices. In conclusion, in section 7 we deal with the challenges to be overcome for successful development of nanobioelectronics.

^e-mail: lak@impb.ru 


\section{Conducting properties of DNA}

The idea that DNA can possess conducting properties was first introduced by Eley and Spivey as early as in 1962 [5]. Even the first experiments on charge transfer in DNA revealed a great variability in the transfer rate. A decisive role in determining the regularities of charge transfer in DNA belongs to the experiments by Giese et al. [6-10]. They demonstrated that current carriers arising in a nucleotide sequence as a result of photoexcitation are holes which hop over guanine bases since the latter have the lowest oxidation potential. For this reason, of special interest for nanoelectronics is the charge transfer in homogeneous or regular nucleotide sequences consisting of G-C nucleotide pairs.

The current voltage characteristic of the conductivity of a homogeneous guanine-cytosine chain was first directly measured in [11]. The authors investigated the electron transport along a Poly G/ Poly C chain of $10.4 \mathrm{~nm}$ length (30 nucleotide pairs) fastened between two electrodes. The measurements were made in the temperature range from room temperature to $4 \mathrm{~K}$. The electric current was detected when the threshold value of the potential difference at the electrodes exceeded $0.5 \div 1 \mathrm{~V}$. This experiment convincingly demonstrated that short homogeneous chains the length of which is less than $10 \mathrm{~nm}$ can carry electrical current.

In paper [12] the capacity of DNA to transfer a charge over a distance of up to $600 \mathrm{~nm}$ was demonstrated. Ohmic behavior of $\lambda$-DNA was also detected in experimental study [13]. In that experiment use was made of a planar geometry in which a DNA molecule was placed onto a mica surface and attached to rhenium-carbon electrodes. The ohmic dependence of the current on the voltage was observed in the range from room temperatures to $1 \mathrm{~K}$. At a temperature below $1 \mathrm{~K}$ an unexpected result was obtained - induced superconductivity arose in DNA.

Numerous experiments on direct measurement of DNA electric conductivity make it clear that DNA has conducting properties.

\section{DNA-based nanowires}

There are different ways to construct nanowires on the basis of DNA. In [14] a technology of creating nanowires from silver was developed in which DNA serves as a scaffold for producing a metal nanowire.

This approach was further developed: the use of DNA as a scaffold enabled one to obtain metal nanowires from $\mathrm{Pd}, \mathrm{Pt}, \mathrm{Cu}, \mathrm{Au}$. A review of recent achievements in this field is given in [15].

Another way to produce nanowires from DNA is to transform DNA from B-form to M-DNA by introducing additional bivalent metals $\left(\mathrm{Zn}^{+2}, \mathrm{Co}^{+2}, \mathrm{Ni}^{+2}\right)$ for $\mathrm{PH}>8.5[16,17]$. It is believed that in the M-DNA metal the ions replace amino-protons of guanines and thymines in each base pair, but the structure can be transformed back to B-DNA by restoring the initial PH level. Recent direct measurements confirmed that M-DNA demonstrates conductivity of metal type and electron transfer is observed in duplexes consisting of 500 base pairs [18]. Apart from ample opportunities of using M-DNA as a nanowire, DNA can be used for constructing biosensors, since it enables one to read out the DNA electron state directly.

\section{Mobility of charge carriers in DNA}

The mobility of the charges in a DNA solution is rather small and corresponds to the case of a smallradius polaron (SRP). In the case of SRP when almost all the charge is localized at one site of the chain, use can be made of the Hamiltonian:

$$
H=\sum_{i} \alpha_{i}|i\rangle\left\langle i\left|+\sum_{i \neq j} v\right| i\right\rangle\langle j|+\sum_{i} \frac{k_{i}}{2} u_{i}^{2}
$$


in which the kinetic energy of the sites can be neglected. Here $\alpha_{i}$ is the energy of a charge at the $i$-th site, $v_{i, j}$ is the matrix element of the transition from the $i$-th site to the $j$-th one, $k_{i}$ is an elastic constant, $u_{i}$ is the displacement of the $i$-th site from the equilibrium position. It is assumed that the charge energy at the site depends linearly on $u_{i}$ :

$$
\alpha_{i}=\alpha_{i}^{0}+\alpha_{i}^{\prime} u_{i}
$$

Let us first consider a homogeneous chain: $\alpha_{i}=0, \alpha_{i}^{\prime}=\alpha, k_{i}=k$ in the approximation of the nearest neighbors $v_{i, j}=v$, for $i=j \pm 1$ and $v_{i, j}=0$, if $i \neq j \pm 1$. A small-radius polaron is formed when either the interaction constant $\alpha^{\prime}$ is large, or the matrix elements of the transition $v$ are small. In this case the total energy of a charge at a site is $E_{i}=\alpha^{\prime} u_{i}+1 / 2 k u_{i}^{2}$ and its minimum is reached for $u_{i}^{0}=-\alpha^{\prime} / k$. Then the potential energy of the charge at the site $U_{i}^{0}$ is equal to $U_{i}^{0}=\alpha^{2} / 2 k$, accordingly, the interaction energy is $V_{i n t, i}^{0}=\alpha^{\prime} u_{i}^{0}=-\alpha^{\prime 2} / k$. Hence, the virial theorem for SRP has the form:

$$
\left|U_{i}^{0}\right|:\left|V_{i n t, i}^{0}\right|:\left|E_{i}^{0}\right|=1: 2: 1 \text {. }
$$

SRP can tunnel to a neighboring site only if the energies and displacements at these sites are equal:

$$
E_{i}=E_{i+1}, \quad u_{i}=u_{i+1} .
$$

Equations (3) have a solution:

$$
u_{i}=-\frac{\alpha^{\prime}}{2 k}=\frac{u_{i}^{0}}{2}
$$

With the use of (4) we can find the activation energy $\Delta E$ of a charge transition to a neighboring site:

$$
\Delta E=E_{i}\left(u_{i}\right)+E_{i+1}\left(u_{i+1}\right)-E_{i}\left(u_{i}^{0}\right)=\frac{\alpha^{\prime 2}}{4 k}=\frac{\left|E_{i}^{0}\right|}{2} .
$$

The SRP mobility can be found using Einstein relation which relates the value of mobility $\mu$ with the diffusion coefficient $D$ :

$$
\mu=\frac{e D}{T}, \quad D=L^{2} k_{t},
$$

where $e$ is the electron charge, $T$ is the temperature, $L$ is the diffusion length, $k_{t}$ is the mean time of the charge occurrence at the site. The expression for $k_{t}$ was obtained by Markus [19], who considered the charge transfer from one site to another as a chemical reaction:

$$
k_{t}=\frac{|v|^{2}}{\hbar} \sqrt{\frac{\pi}{E_{r} T}} e^{-E_{r} / 4 T}
$$

where $E_{r}$ is the so-called reorganization energy which in our model has a simple form: $E_{r}=4 \Delta E$.

In the case of an inhomogeneous chain, formula (7) can easily be modified. For example, when there is a donor and an acceptor joined by a bridge, as in the case of a polynucleotide chain Poly $G A_{1} \ldots A_{k-1} G /$ Poly $C T_{1} \ldots T_{k-1} C$ with $k>2$, in formula (7) we should use instead of $v$ its value obtained in the super exchange theory:

$$
v=\frac{v_{G A} v_{A G}}{\Delta E_{A G}}\left(\frac{v_{A A}}{\Delta E_{A G}}\right)^{k-2},
$$

where $v_{G A}$ and $v_{A G}$ are matrix elements of the transition from guanine to adenine and from adenine to guanine, respectively, $v_{A A}$ is the matrix element of the transition between adenines, $E_{A G}$ is the difference of energies on the adenine and guanine. 
As shown in [20], for the DNA parameters used in this work which correspond to an intermediate coupling, an increase in the hole mobility which takes place when the temperature decreases, will be replaced by its decrease according to formulas (6)-(7).

The obtained results can be generalized to the case of variable range hopping when electron transfers from $\mathrm{i}$-th to $\mathrm{i}+\mathrm{R}$ site. In this case $v \approx \exp (\alpha R)$, where $\alpha$-is the constant which defines the wave function overlapping between $i$-th and $(i+R)$-th site. It follows from (6), (7) that:

$$
\mu \approx \exp \left(-2 \alpha R-\frac{E_{r}}{4 T}\right)
$$

According to Mott:

$$
E_{r} \cong \frac{c}{R N_{F}}
$$

and the optimal $R$ will be equal to: $R=\sqrt{c / 8 \alpha N_{F} T}$, i.e., immediately lead us to the expression: $\mu \approx \exp \left[-\left(T_{1} / T\right)^{1 / 2}\right]$, where $T_{1}=2 c / N_{F} \alpha$.

\section{Electronic nanobiochip}

One of the actively progressing areas concerned with DNA/RNA analysis is the development of DNAbases biochips. Since the time of creation of first DNA-based chips [21] they have became a powerful tool for the investigation of the living systems. The overwhelming majority of DNA-based biochips are optical chips. Recently, however, increasing attention has been given to electronic biochips which require no special marking. These biochips measure the electric current induced by the presence of a biomacromolecule being detected [22]. Such great attention to nanobiochips is due to the huge potential advantages which they can offer: diminutiveness (up to nano size scales), small time required for the detection, lack of necessity to use markers, low price, etc.

Though the development of such chips is in its initial stage it is expected that they will be able to detect even trace concentrations of biomolecular targets, up to single molecules.

Leaving aside construction details, in terms of the principle of detecting, most of the biochips can be divided into optical and electrical.

The principle scheme of the design and operation of an optical biochip is as follows. On a template (which may be glassy, plastic, silicon, etc.) single-strand oligonucleotides of a special type (i.e. with a known nucleotide sequence) are tied up as individual spots. Each spot contains oligonucleotides of only one type. The size of a spot is from $20 \mu \mathrm{m}$ to $100 \mu \mathrm{m}$. The number of spots on a template may be as great as tens of thousands, i.e. one chip can perform diagnosis of tens of thousands of genes at a time.

For the optical biochips, there is a physical limitation on the number of spots on a chip because the size of one spot must be not less than the wavelength of the light, i.e. $\approx 1 \mu \mathrm{m}$. Passing on from optical methods to electrical ones in this case is caused by simplification, quickness, low cost, etc. of diagnostics in the latter case [23-27].

\section{Electronic memory devices}

The progress in nanoelectronics has led to the development of actual molecular memory devices. Thus, in papers $[28,29]$ an actual chip of operative memory with a density of $7 \cdot 10^{9} \mathrm{bit} / \mathrm{cm}^{2}$, is developed which is formed by blocks of 64 bits with a density of $5 \cdot 10^{11} \mathrm{bit} / \mathrm{cm}^{2}$ in the block on the basis of rotaxane molecules. These molecules are constructed from fragments devoid of chemical bonds and capable of moving relative to one another. The rotaxane molecules have two stable states 
which can alternate between each other when placed in an electric field. A matrix architecture was used with a system of parallel metal electrodes $\approx 10 \mathrm{~nm}$ in thickness placed onto the template. A monolayer of rotaxane molecules was formed on these electrodes. The upper part of the rotaxane molecules was attached to the upper part of metal electrodes arranged perpendicularly to the lower ones. It should be noted that the storage density on the rotaxanes is nearly two orders of magnitude higher than the density in currently produced devices $\left(\approx 10^{8} \mathrm{bit} / \mathrm{cm}^{2}\right)$.

In [28], an electronic memory was developed on the basis of the tobacco mosaic virus. For this purpose the authors also used a matrix architecture with the diameter of aluminium nanowires $\approx 30$ $\mathrm{nm}$. At the nodes of these nanowires tobacco mosaic viruses covered by platinum nanoparticles $\approx 10$ $\mathrm{nm}$ in diameter were placed. In view of the fact that the viruses were dispersed chaotically into a polyvinyl spirit matrix which formed a sandwich structure between the electrodes, the nodes connected by hybrid platinum-virus electrodes were also arranged chaotically. For storage, use was made of the hysteresis in the current-voltage characteristic of viruses modified by platinum nanoparticles. The memory device obtained is actually the first hybrid biomolecular device of nanometer size.

The presumed DNA-based electronic memory is similar in its arrangement to the memory on the basis of rotaxanes or tobacco mosaic viruses [30]. The principle of operation of such a memory is based on hysteresis of the conducting properties of short DNA duplexes depending on the electric field value [11]. Address encoding and decoding of information is realized by matrix way. As distinct from a biochip, in creating a memory device use is made not of single-strand oligonucleotides, but of their duplexes. The storage density in such a memory can be $10^{6} \div 10^{9}$ times larger than in modern integrated circuits.

\section{Conclusions}

Different instances of the use of DNA in nanoelectronics have been considered. Some of the described devices are already realized as pilot samples, others can be developed in the near future. The examples given convincingly demonstrate the possibility to create the analog of any solid-state electronic device on the basis of biomacromolecules. The advantages of biomacromolecules in the nanometer size range are obvious. One of the main requirements of the modern electronics is the copying ability, i.e., the development of a huge number of similar elements. This requirement will hardly be met at the nanometer scale. For example, for such a promising material as nanotubes, it is difficult to copy exactly their size and chirality, which determine their intrinsic electronic properties. Another challenge is the development of heterostructures to be used in nanodevices which would contain nanotubes as their elements.

The main difficulty of the use of biomacromolecules in nanoelectronics is the absence of a technology comparable in efficiency to that used in producing solid-state electronic devices. Actually, the first steps towards the development of such a technology are being taken. For this reason for the development of nanobiochips and, more broadly, for the development of nanobioelectronics, what is needed above all is the conduction of experiments at the molecular level, construction at a molecular level, manipulation by individual molecules. Without developing a technology of manipulation at a molecular level one cannot construct electronic devices of molecular scale. These technologies are not straightforwardly associated with the construction of any particular device - development of such technologies should acquire the status of applications. An important role in the development of nanobioelectronics and construction of nanobiochips should belong to theoretical investigations. Such investigations always precede the construction of a device in practice. They should be focused on the problems without which creation of a molecular electronic device is practically impossible. Construction of nanobiochips and nanobioelectronic devices on the basis of theoretical calculations and experimentally developed methods is the main aim of nanobioelectronics. 


\section{Acknowledgements}

The work was supported by RSF project N 16-11-10163.

\section{References}

[1] A. Dragon, Sci. Am. 5, 112 (1998)

[2] E. Winfree, F. Liu, L.A. Wenzler, N.C. Seeman, Nature 394, 539 (1998)

[3] N.C. Seeman, Sci. Amer. 290, 64-75 (2004)

[4] E. Braun, Y. Eichen, U. Sivan, G. Ben-Yoseph, Nature 391, 775 (1998)

[5] D.D. Eley, D.I. Spivey, Trans. Faraday Soc. 58, 411-415 (1962)

[6] E. Meggers, M.E. Michel-Beyerle, B. Giese, J. Am. Chem. Soc. 120, 12950-12955 (1998)

[7] B. Giese, S. Wessely, M. Spormann, U. Lindeman, E. Meggers, M.E. Michel-Begerle, Angew. Chem. Int. Ed. 38, 996-998 (1999)

[8] M. Bixon, B. Giese, S. Wessely, T. Langenbacher, M.E. Michel-Beyerle, Jortner J., PNAS 96, 11713-11716 (1999)

[9] B. Giese, J. Amaudrut, A.K. Köhler, M. Spormann, S. Wessely, Nature 412, 318-320 (2001)

[10] B. Giese, Acc. Chem. Res. 33, 631-636 (2000)

[11] D. Porath, A. Bezryadin, S. de Vries, C. Dekker, Nature 403, 635 (2000)

[12] H.W. Fink, C. Schönenberger, Nature 398, 407 (1999)

[13] A.Y. Kasumov, M. Kociak, S. Gueron et al., Science 291, 280 (2001)

[14] E. Braun, Y. Eichen, U. Sivan, G. Ben-Yoseph, Nature 391, 775 (1998)

[15] Q. Gu, C. Cheng, R. Gonela et al., Nanotechnology 17, R. 14 - R. 25 (2006)

[16] J.S. Lee, J.P. Latimer, R.S. Reid, Biochem. Cell. Biol. 71, 162-168 (1993)

[17] S.W. Lee, C. Mao, C.E. Flynn, A.M. Belcher, Science 296, 892-895 (2002)

[18] A. Rakitin, P. Aich, C. Papadopoulos, Y. Kobzar et al., Phys. Rev. Lett. 86, 3670 (2001)

[19] R.A. Marcus, Rev. Mod. Phys. 65, 599 (1993)

[20] V.D. Lakhno, Int. J. Quant. Chem. 108, 1970 (2008)

[21] A.D. Mirzabekov, Vestnik RAS 73, 412-421 (2003)

[22] J.B. Rampal (Ed.), Microarrays. Volume I: Synthesis Methods (Series: Methods in Molecular Biology, N 381, Humana Press, Totowa, New Jersey, USA, 2007) 469 p.

[23] G. Marchand, C. Delattre, R. Campagnolo, P. Pouteau, F. Ginot, Analytical Chem. 77, 51895195 (2005)

[24] J.J. Gooding, Electroanalysis 14, 1149-1156 (2002)

[25] E. Palecek, F. Jelen, Crit. Rev. Anal. Chem. 3, 261-270 (2002)

[26] J. Wang, Anal. Chim. Acta 469, 63-71 (2002)

[27] T.G. Drummond, M.G. Hill, J.K. Barton, Nature biotechnology 21, 1192-1199 (2003)

[28] Y. Luo, C.P. Collier, J.O. Jeppesen et al., ChemPhysChem 3, 519-525 (2002)

[29] J.E. Green, J.W. Choi, A. Boukai et al., Nature 445, 414-417 (2007)

[30] R.J. Tseng, C. Tsai, L. Ma, J. Ouyang et al., Nature nanotechnology 1, 72-77 (2006) 